\title{
Industry training on on-wafer optoelectronic vector network analysis
}

Nicholas H. L. Wong, Brian Pile, Junqiang Zhou

Nicholas H. L. Wong, Brian Pile, Junqiang Zhou, "Industry training on onwafer optoelectronic vector network analysis," Proc. SPIE 11143, Fifteenth Conference on Education and Training in Optics and Photonics: ETOP 2019, 1114312 (2 July 2019); doi: 10.1117/12.2523481

Event: Fifteenth Conference on Education and Training in Optics and Photonics: ETOP 2019, 2019, Quebec City, Quebec, Canada 


\title{
Industry training on on-wafer optoelectronic vector network analysis
}

\author{
Nicholas H. L. Wong ${ }^{\mathrm{a}}$, Brian Pile ${ }^{\mathrm{b}}$, and Junqiang Zhou ${ }^{\mathrm{a}}$

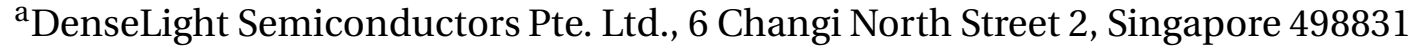 \\ ${ }^{b}$ POET Technologies Inc., 780 Montague Expressway, Suite 107, San Jose, CA 95131, USA
}

\begin{abstract}
In today's fast-paced world, efficient industrial workplace training has economic implications in any technology firm. Employees are required to learn technical skills and acquire proficiency quickly, regardless of the extent of prior experience. In this paper, we outline a methodology to train technical staff, which could include engineers, technicians, and/or operators, on vector network analysis in the context of photonics companies involved in the characterization of optoelectronic devices, particularly photonic integrated circuits. The acquired skills span electronic, microwave, and optical domains, but assume only basic prior electrical knowledge and no microwave or optics background. Focusing on on-wafer $S$-parameter characterization of optoelectronic components or modules, we first describe training in various laboratory tasks, including instrument calibration, device handling, probing, optical waveguide alignment, and taking measurements. Then, we elaborate on some relevant skills for processing and analyzing measured data. We identify relevant theory where appropriate, in a way suited for industry needs as opposed to academic settings. In this manner, staff learn sufficiently to be able to level their skills swiftly to get the job done. Much of the pedagogical details stem from our own industrial experiences as a photonic wafer manufacturing company, where we have trained team members of various technical backgrounds. The pedagogy can be applied to other technical areas, and it is hoped that the ideas in this paper will be of value to both nascent and established organizations.
\end{abstract}

Keywords: Optoelectronics, Vector network analysis, S-parameters, Photonic integrated circuit, On-wafer, Photonics education, Industry training, Teaching methodologies.

\section{INTRODUCTION}

In both radio-frequency (RF) and photonics disciplines, the measurement and analysis of scattering parameters ( $S$ parameters) are of great importance in studying device behavior. It behooves industry firms to efficiently educate new staff to quickly develop necessary skills and maintain productivity. However, this nontrivial subject is typically first encountered only at senior undergraduate levels. Although work has been done to improve $S$-parameter education, these remain largely academic, ${ }^{1,2}$ requiring coverage of advanced concepts like Smith charts, or include extensive circuit analysis. ${ }^{3}$ On the other hand, most operators are not college graduates, let alone possess any RF knowledge, and not all engineers have formal RF education.

In the absence of such prior technical exposure, there is a need to train new staff on how to perform $S$-parameter measurements, which we call vector network analysis, in an efficient manner. This paper seeks to address this by outlining industry training activities for various practical procedures, in the case of on-wafer measurements of optoelectronic devices. While both RF and optics concepts are covered, no prior knowledge in these domains is assumed.

Whereas engineers might be able to self-learn, proper training of operators is vital, as insufficient training can result in wasted production time and other inefficiencies. Furthermore, it has been shown that educating operators with basic technical fundamentals, as opposed to mere procedural instruction, enhances their knowledge and enables them to be less reliant on engineers when troubleshooting becomes necessary. ${ }^{4}$

Sec. 2 gives a background of the device used as an example in this paper, as well as the measurement set-up. Sec. 3 establishes the general training methodology. Key laboratory tasks and analysis skills are described in Secs. 4 and 5, respectively. Finally, Sec. 6 concludes the paper.

Further author information: (Send correspondence to N.H.L.W.)

N.H.L.W.: E-mail: n.hl.wong@ieee.org

Fifteenth Conference on Education and Training in Optics and Photonics: ETOP 2019, edited by

Anne-Sophie Poulin-Girard, Joseph A. Shaw, Proc. of SPIE Vol. 11143, 111431Z · (c) 2019

SPIE, ICO, IEEE, OSA · CCC code: 0277-786X/19/\$18 · doi: 10.1117/12.2523481

Proc. of SPIE Vol. 11143 111431Z-1 


\section{BACKGROUND}

We consider an example optoelectronic device-under-test (DUT) shown in Fig. 1. It is a photonic integrated circuit comprising a waveguide and a photodetector. The waveguide, whose entry facet is cleaved and anti-reflection coated to maximize coupling, guides external light to the photodetector. The optical signal causes the photodetector to produce a corresponding electronic signal (a photocurrent), which can be probed via the Ground-Signal-Ground (GSG) pads.

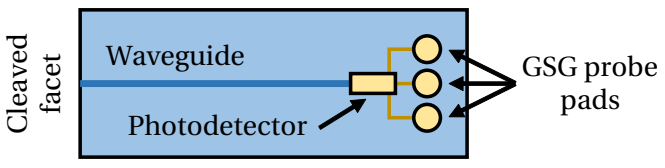

Figure 1. Photonic integrated circuit DUT consisting of waveguide, photodetector, and probe pads.

The DUT has a low-pass frequency response, and the objective is to measure its optoelectronic 3-dB bandwidth $f_{3 \mathrm{~dB}}$, defined as the frequency at which the magnitude response drops by $3 \mathrm{~dB}$ of its peak value at direct current (DC). To do so, the set-up in Fig. 2 is used. The main instrument is the vector network analyzer (VNA), which measures $S_{21}$ (among other $S$-parameters), from which $f_{3 \mathrm{~dB}}$ can be extracted. An optical input is provided by the laser diode. This is modulated by the Mach-Zehnder modulator (MZM), whose modulation signal is supplied by the VNA at Port 1. The modulated optical signal is then divided by a $95: 5$ splitter. The $5 \%$ arm goes to an optical power meter, while the $95 \%$ main arm goes to a cleaved or lensed fiber patchcord mounted onto a V-groove on a translation stage. This is buttcoupled to the waveguide input of the DUT, which is also situated on a translation stage. The power meter reading is used to aid in fiber alignment.

The DUT performs an optical-to-electrical $(\mathrm{O} / \mathrm{E})$ signal conversion, which is sensed by the RF probe in contact with the DUT's electrical pads. This RF signal is then fed back to the VNA via Port 2. A source meter is used to supply a bias voltage to the DUT, through the VNA's Port 2.*

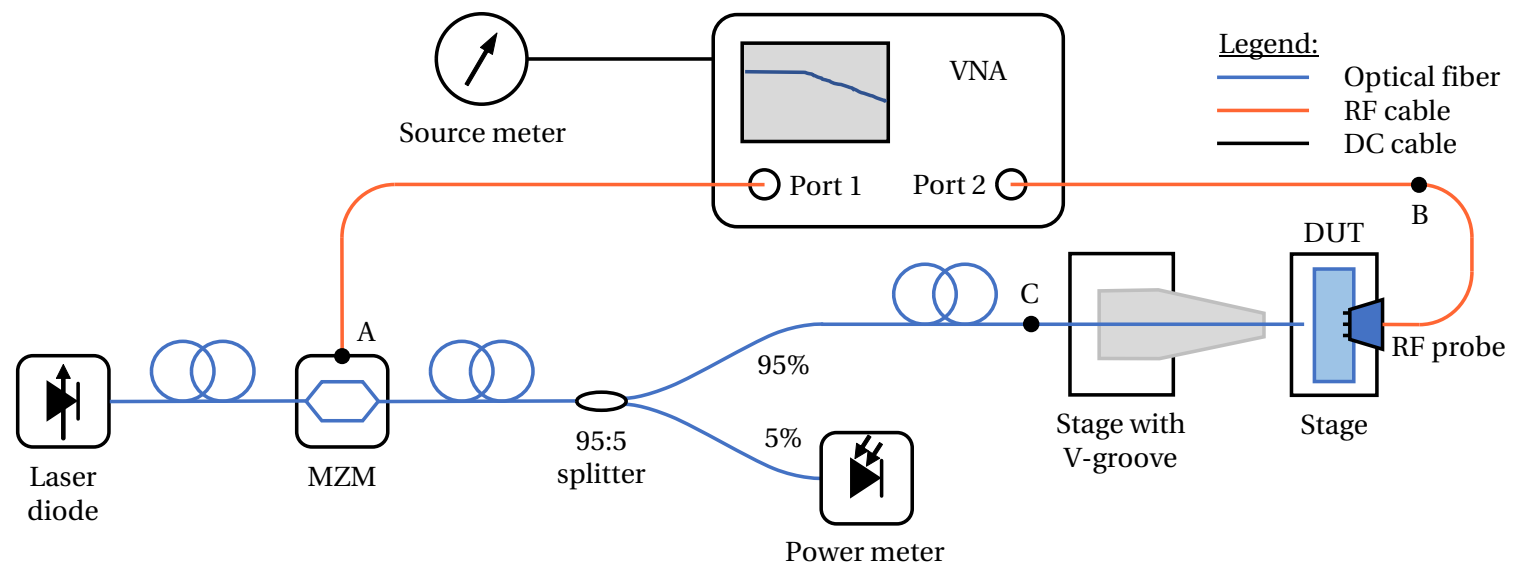

Figure 2. O/E S-parameter measurement set-up.

Through sufficient training, both engineers and operators are able to perform the required network analyzer measurements. We note that component sourcing and assembly are outside the training scope; the procedures described in the subsequent sections assume a fully assembled set-up.

\section{METHODOLOGY}

In order to enhance the learning process, trainees experience multiple learning modalities. ${ }^{5,6}$ Applying the VARK model, ${ }^{7}$ these include:

\footnotetext{
${ }^{*}$ The VNA has an internal bias tee which combines RF and DC signals.
} 
- Visual: Trainees observe a trainer demonstrating how to perform tasks.

- Auditory: Trainees listen to the explanations given by trainers.

- Read/write: Trainees read through operational procedure manuals. They are also encouraged make their own notes for self-reference.

- Kinesthetic: After the above introductory familiarizations, trainees gain hands-on experience in performing the tasks under supervision.

In all of the various tasks described in the following sections, training is conducted through the overarching process shown in Fig. 3. Any new task is introduced by a trainer (an experienced engineer or technician); trainees observe, listen, and take notes. Thereafter, trainees personally perform the task while under trainer supervision. This may be repeated a number of times for trainees to gain hands-on experience and for the trainer to correct any mistakes iteratively. After no more mistakes are observed, the trainer lets trainees perform the task without supervision in order to build confidence. Again, this may go through a few repetitions. Once the trainer is satisfied that the trainees can perform the task correctly and independently, the latter are deemed qualified.

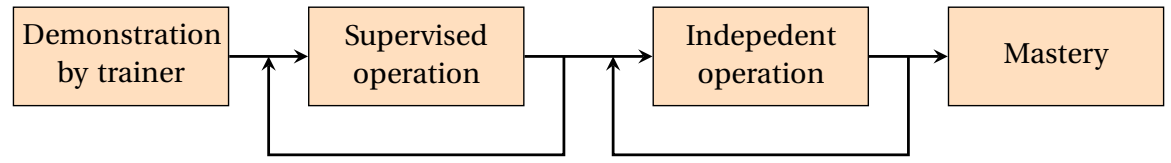

Figure 3. Practical training process.

\section{LABORATORY TASKS}

This section describes tasks and training that are needed to conduct optoelectronic vector network analysis. Various tactile tasks are first described. Subsequently, the critical tasks of instrument calibration and the actual measurements are explained in detail.

\subsection{Tactile Tasks}

These tasks relate to general wafer and DUT manipulations. Operators need to perform them on a regular basis and so supervised training is conducted for them. On the other hand, engineers, needing to possess the related skills for ocassional work, learn through self-practice.

\subsubsection{Device Handling}

Being in a wafer manufacturing organization, one the first things that new operators get trained to do is the proper handling of wafers, which can be down to $\varnothing 2$ in. More critically, they are trained to manually handle 'cells' or 'bars' that have been cleaved out from the initial wafers. These can have dimensions down to the $100 \mathrm{~s}$ of $\mu \mathrm{m}$ and thicknesses down to the 100s of nm.

Operators are shown proper wafer and bar handling by an engineer or supervising technician, then typically spend a few days to a week practicing various hands-on manipulations, including picking, placing, transferring, flipping, and rotating. This repetitive practice enables them to develop the familiarity and muscle memory needed to know

- What tool to use to pick wafer pieces.

- How much pressure to apply when holding pieces by tweezer.

- How to maintain grip and not drop pieces in transit.

- Which storage option to use, e.g. photon ring or gel pack, and how to remove or emplace pieces on or in them.

Another key skill is the ability to manipulate and position DUTs on translation stages while using microscopes for visual navigation. Whereas introductions to instrument usage and the availability of objectives of different magnifications are important, users ultimately gain experience in this task through repeated hands-on practice. 


\subsubsection{RF Probing}

As illustrated in Fig. 4, each DUT has electrical pads for probing. Operators are trained on how to maneuver the RF probe safely. This includes probe positioning, probe lowering, and visually recognizing when contact is made.

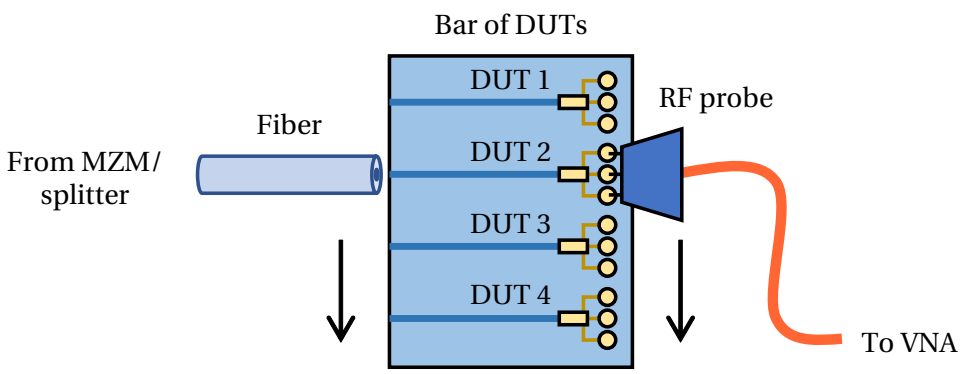

Figure 4. DUT in contact with probe and with aligned fiber. Arrows indicate direction of sequential measurements.

\subsubsection{Optical Waveguide Alignment}

Operators are trained on aligning the fiber to the DUT's waveguide. They develop a 'feel' for moving the fiber along the stage's three dimensional axes, while maximizing some proportional indicator, be it an optical power meter reading or a source meter photocurrent reading. They also learn fiber cleaving, and how to recognize when recleaving is needed.

\subsection{Instrument Calibration}

A fully assembled measurement set-up, as in Fig. 2, includes inline components, cables, and adapters external to the VNA. These influence the responses measured from the DUT, in the form of magnitude and phase changes. The basic purpose of calibration is to account for all these sources and subtract their influence from the measurement, so that the calibrated response can be attributed solely to the DUT.

Calibration is always done before measurements and engineers and operators need careful mastery of this crucial step to ensure that data is meaningful. Sec. 4.2.1 explains some theory that engineers will find helpful to understand the origins of special frequency response cases for probe calibration. Whereas operators need not know the theory, they must still be aware of what the response profiles look like. Sec. 4.2.2 then outlines the calibration procedure.

\subsubsection{S-Parameter Theory}

Vector network analysis involves the measurement of the scattering parameters or S-parameters of linear electrical or optical networks. In the simplest case, a circuit or DUT can be represented by a 1- or 2-port network, as in Fig. 5. Each port is an input (subscript ${ }_{1}$ ) or output (subscript ${ }_{2}$ ) and has a pair of terminals, which interface with the incident $(a)$ and reflected $(b)$ power waves. ${ }^{8}$
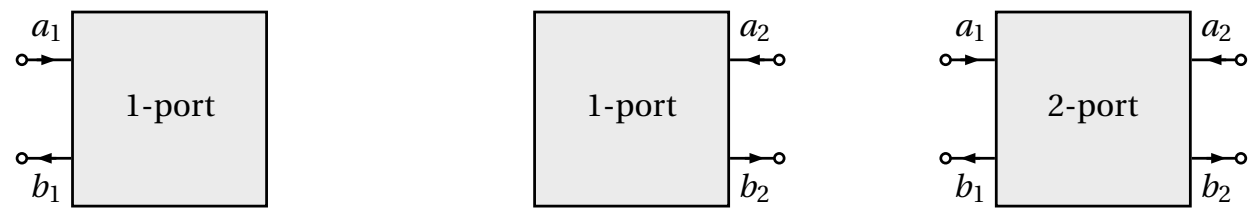

Figure 5. 1-port and 2-port networks.

The interactions of these waves can be consolidated into a scattering matrix given by

$$
\left(\begin{array}{l}
b_{1} \\
b_{2}
\end{array}\right)=\left(\begin{array}{ll}
S_{11} & S_{12} \\
S_{21} & S_{22}
\end{array}\right)\left(\begin{array}{l}
a_{1} \\
a_{2}
\end{array}\right)
$$

where the $S$-parameters are the elements of the matrix: 
- $S_{11}=\left.\frac{b_{1}}{a_{1}}\right|_{a_{2}=0}=\Gamma_{\text {in }}$ is the reflection coefficient at the input port,

- $S_{21}=\left.\frac{b_{2}}{a_{1}}\right|_{a_{2}=0}=G_{\mathrm{fwd}}$ is the forward transmission (gain or loss) coefficient,

- $S_{12}=\left.\frac{b_{1}}{a_{2}}\right|_{a_{1}=0}=G_{\text {rev }}$ is the reverse transmission coefficient,

- $S_{22}=\left.\frac{b_{2}}{a_{2}}\right|_{a_{1}=0}=\Gamma_{\text {out }}$ is the reflection coefficient at the output port.

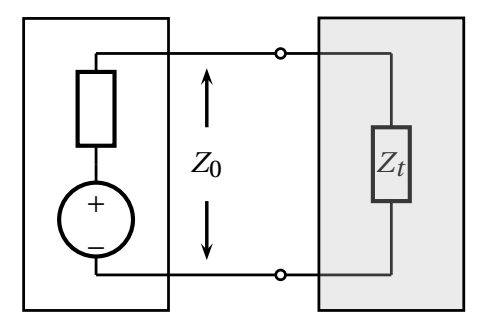

Figure 6. 1-port network with source.

As will be evident, 1-port networks deserve further elaboration. Fig. 6 depicts a 1-port network (grey box), represented by a load or termination with impedance $Z_{t}$, connected to a source (white box). The source, represented by an ideal source in series with an impedance, is assumed to be matched to the characteristic impedance $Z_{0}$ of the transmission lines connecting it and the network. From most RF electronics textbooks, ${ }^{8}$ it is easy to show that the reflection coefficient, $\Gamma=S_{11}$ or $S_{22}$, is given by

$$
\Gamma=\frac{Z_{t}-Z_{0}}{Z_{t}+Z_{0}}
$$

This is generally a complex quantity, since $Z_{t}$ can include reactances from capacitors and/or inductors in the network. We can express $\Gamma$ in polar form

$$
\Gamma=\rho \mathrm{e}^{\mathrm{j} \phi},
$$

where $\rho=|\Gamma|$ is the magnitude, $\phi=\angle \Gamma$ is the phase, e is Euler's number ( $\mathrm{e}^{x} \equiv \exp x$ ), and $\mathrm{j}=\sqrt{-1}$ is the imaginary unit.

During calibration of network analyzers with RF probes, measurements of networks with known special termination cases, namely, open circuit (OPEN), short circuit (SHORT), and matched load (LOAD), are needed. At radio frequencies, the behaviors of these networks are frequency-dependent and deviate from their DC behavior. The OPEN, SHORT, and LOAD cases can be modeled as a capacitance $C_{t}$, an inductance $L_{t}$, and an inductance $L_{t}$ in series with a resistance $R_{t}$, respectively, ${ }^{9}$ as depicted in the circuit diagrams in Tab. 1 . The table also shows the formulae for calculating the impedance $Z_{t}$ of the respective terminations, where frequency $f$ and angular frequency $\omega$ are related by $\omega=2 \pi f$. Examples of frequency responses are also shown. These were obtained via circuit simulation (Sim) using the parameter values of a particular Cascade Microtech probe. Their shapes can be understood by considering each case:

- Open circuit: Modeled as a small capacitance $C_{t}$. All power is reflected, so $\rho=|\Gamma|=1=0 \mathrm{~dB}$ for all $f$. At DC, $Z_{t}=\infty$; from Eqs. (2) and (3), $\Gamma=1=(1) \mathrm{e}^{\mathrm{j} 0} ;\left.\therefore \phi\right|_{f=0}=0^{\circ}$. At high $f, Z_{t}=1 /\left(\mathrm{j} \omega C_{t}\right) \rightarrow 0 \Omega$ and the termination tends towards a SHORT; $\lim _{f \rightarrow \infty} \phi=-180^{\circ}$.

- Short circuit: Modeled as a small inductance $L_{t}$. All power is reflected, so $\rho$ is always $0 \mathrm{~dB}$. At DC, $Z_{t}=0 \Omega ; \Gamma=$ $-1=(1) \mathrm{e}^{\mathrm{j} 180} ;\left.\therefore \phi\right|_{f=0}=180^{\circ}$. At high $f, Z_{t}=\mathrm{j} \omega L_{t} \rightarrow \infty$ and the termination tends towards an OPEN; $\lim _{f \rightarrow \infty} \phi=0^{\circ}$.

- Matched load: Modeled as a small inductance $L_{t}$ in series with the load $R_{t}=Z_{0}$. At DC, all power is absorbed, so $\rho=|\Gamma|=0=-\infty \mathrm{dB}$ according to Eq. (2); $\left.\phi\right|_{f=0}=0^{\circ}$ and at positive frequencies very close to $0 \mathrm{~Hz}, \lim _{f \rightarrow 0^{+}} \phi=90^{\circ}$ (hence, the rapid $0^{\circ}$ to $90^{\circ}$ jump). At high $f$, the impedance of $L_{t}$ dominates over $R_{t}$ and the behavior mimics that of the SHORT (inductor only) termination; $\lim _{f \rightarrow \infty} \rho=0 \mathrm{~dB}, \lim _{f \rightarrow \infty} \phi=0^{\circ}$.

Knowledge of these ideal frequency response profiles is useful for assessing calibration quality. 
Table 1. Frequency responses of 1-port network terminations. The values of $C_{t}$ and $L_{t}$ are for a particular I40-GSG-75 Cascade probe.

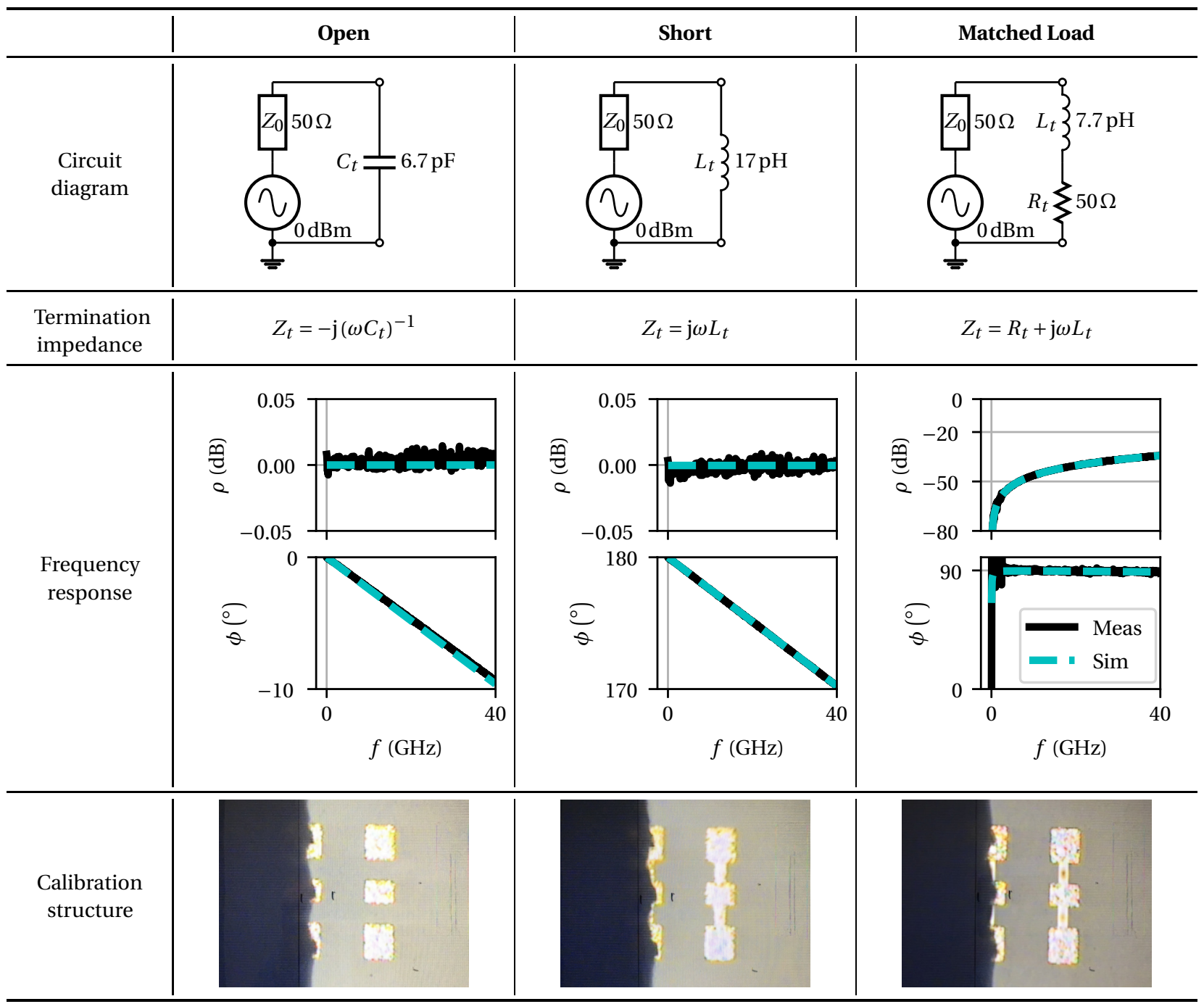

\subsubsection{Calibration Procedure}

We refer to the set-up in Fig. 2. Calibration can be understood by systematically considering each 'component' in terms of electrical $(E)$ or optical $(O)$ planes and segments. The two VNA ports are the reference $E$ interfaces, denoted as the 'VNA plane'. Between these, the interface segments can be simplified to be $\mathrm{E}-\mathrm{E} / \mathrm{O}-\mathrm{O} / \mathrm{E}-\mathrm{E}$. The first $\mathrm{E}-\mathrm{E}$ segment is the cable connection between Port 1 and point $A$ at the MZM's RF input. The E/O segment, between points A and C, comprises the optical components of the MZM, splitter, and fiber patch cord. ${ }^{\dagger}$ The O/E segment is the DUT itself. Hence, this is specifically an $\mathrm{O} / \mathrm{E}$ measurement. Finally, the last $\mathrm{E}-\mathrm{E}$ segment consists of two parts: the 'probe assembly' comprising the RF probe and RF cable up to point B, and the RF cable continuing to the VNA's Port 2 . We choose an interface at point $\mathrm{B}$ so as not to mechanically disturb the probe during the necessary cable manipulation in the calibration step. The end result of calibration is that the $E-E$ and $E / O$ segments are canceled out, leaving only the O/E response of the DUT.

Fig. 7 outlines the calibration procedure. A preset is first done to initialize the VNA and remove any previously

\footnotetext{
${ }^{\dagger}$ Ideally, the V-groove-mounted bare fiber should also be included. However, its loss is assumed to be negligible, so we can consider the patch cord's FC connector as the E/O plane termination.
} 
cached calibrations. We then begin by connecting an AutoCal module ${ }^{\ddagger}$ between points A and B and performing a 2port calibration, saving the calibration 'setup' file in the process. This effectively cancels out the RF cable effects and shifts the VNA plane to A and B. Without any connection changes, a 1-port calibration is also done at B to obtain a setup file for later use. The subsequent step is to account for the $E / O$ segment. This $E-?-O / E-E$ configuration requires a known $\mathrm{O} / \mathrm{E}$ response to be used as a reference. Such is obtained by using a vendor-provided $\mathrm{O} / \mathrm{E}$ calibration module (CalMod), in place of the DUT. The RF connections are made to the MZM input and O/E CalMod output at A and B, respectively. The splitter's output fiber is also connected to the O/E CalMod's optical input, and a measurement is made to obtain the $E / O$ segment's response in a Touchstone . $s 2 \mathrm{p}$ file.

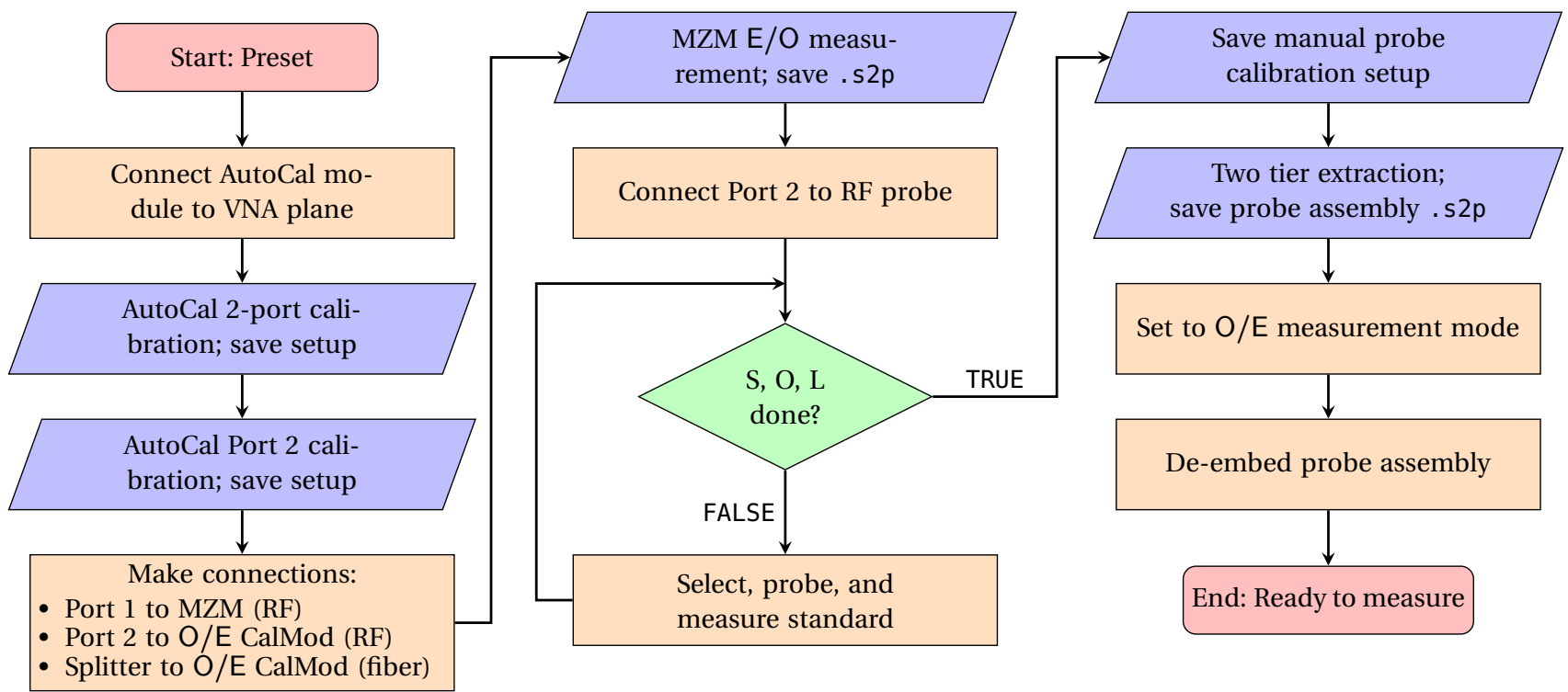

Figure 7. Calibration procedure.

Next, the probe assembly needs to be de-embedded. ${ }^{10}$ In this 1-port calibration, the VNA's Port 2 is connected via point B to the RF probe. Using the SHORT-OPEN-LOAD (SOL) technique, ${ }^{11}$ probe measurements of short circuit, open circuit, and matched load standards on an impedance standard substrate (ISS) are done. In an iterative manner, each standard is manually probed, as shown in the calibration structure microscope images in Tab. 1, and measured, until all three are done and a setup file is created. At this stage, it is beneficial to manually re-measure each standard to check the calibration, given the probe's sensitivity to parasitics and environmental perturbations. Sample measured (Meas) frequency responses of each standard are plotted in Tab. 1 against their simulated counterparts. It is at this juncture that knowledge of the expected responses is important. In the presence of noise and other inaccuracies, engineers and operators are trained to assess a calibration as 'good' if these conditions are satisfied:

- OPEN: $|\rho| \leq 0.5 \mathrm{~dB} ;\left.\phi\right|_{f=0}=0^{\circ}$.

- SHORT: $|\rho| \leq 0.5 \mathrm{~dB} ;\left.\phi\right|_{f=0}=180^{\circ}$.

- LOAD: $\rho \leq 20 \mathrm{~dB} ;\left.\phi\right|_{f=0}=90^{\circ}$.

Unless a satisfactory probe calibration is achieved, it is repeated. Afterwards, through the VNA's software menu, a twotier calibration ${ }^{9}$ is done, using the setup files of the probe and the Port 2 AutoCal, to calculate the probe assembly's influence in a Touchstone file. Finally, the VNA is set to O/E measurement mode, in which the software factors in the VNA plane and $\mathrm{E} / \mathrm{O}$ calibration files. The probe assembly is de-embedded and the calibration is complete.

At this point, a measurement of a 'golden sample' DUT is taken. This sample has a prior reference measurement that was taken after a past good calibration. A visual comparison is done between the current and previous measurements

\footnotetext{
${ }^{\ddagger}$ Alternatively, a mechanical calibration kit can be used.
} 
(e.g. $S_{21}$ magnitude) and a judgement is made on their relative similarity to decide if the calibration is indeed valid or should be repeated.

Whereas engineers may be quicker to internalize the procedure by grasping the logic of the steps, operators can gain familiarity too, albeit by means of extended engineer supervision, memory work, and repeated practice. A step-by-step manual, including photographs and menu selection screenshots, is helpful.

\subsection{On-Wafer Measurements}

After successfully calibrating the set-up in Fig. 2, DUT measurements can proceed. The procedure is charted in Fig. 8. Firstly, the DUT is appropriately positioned on the translation stage within simultaneous contact and alignment ranges of the probe and fiber, respectively. Device handling and microscope navigation skills (Sec. 4.1.1) are needed here. Secondly, the probe is lowered to make contact with the DUT as per Sec. 4.1.2 and Fig. 4, where multiple DUTs in the same bar are to be measured sequentially. Thirdly, the bias voltage $V_{\text {bias }}$ is set on the source meter and enabled. The source meter (in 'current sense' mode) also measures the DUT's generated photocurrent $I_{p}$. Upon enabling $V_{\text {bias }}$, an observed, albeit small, increase in $\left|I_{p}\right|$ suggests good probing; this corresponds to the dark current. Conversely, no change is a telling indicator of inadequate probe contact. Alternatively, the source meter set to 'voltage sense' mode would register at compliance (i.e. open circuit) for poor contact.

Next, the laser is enabled. The reading on the power meter is used to monitor the integrity of the optical connections, while the main stage-mounted fiber arm is aligned to the DUT's waveguide input (see Sec. 4.1.3 and Fig. 4) by maximizing the $\left|I_{p}\right|$ reading on the source meter. After a good alignment is achieved, a discernible low-pass-filter-like trace should be visible on the VNA's user interface, if it is continuously sweeping and set to display $\left|S_{21}\right|$.

Finally, sweep averaging is activated on the VNA if required, and the measurement is recorded and saved into a Touchstone file. Users are trained to follow specific filenaming protocols. The process is then repeated for subsequent DUTs until the test lot is completed.

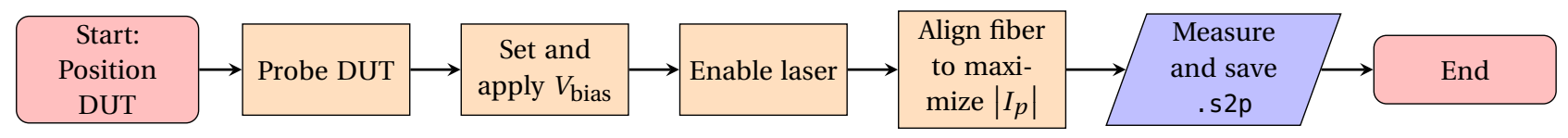

Figure 8. Measurement procedure.

Based on observations and verbal feedback, we found that operators can master the measurement procedure much more quickly and confidently than the calibration procedure. This is likely due to a few reasons. Firstly, the measurement procedure is conspicuously shorter and more linear. Secondly, while both procedures include numerous kinesthetic actions, the measurement steps involve manipulations of more 'real-world' objects (e.g. probing the DUT or aligning the fibre), as opposed to clicking through the more 'virtual' calibration and de-embedding software menus.

\section{ANALYSIS SKILLS}

Taking measurement data is only part of the story; the data also needs to be analyzed to discover behaviors and draw conclusions. Pertaining mainly to engineers, these skills assume some existing knowledge of the DUT design and process parameters.

\subsection{Scripting}

In the wafer design process, numerous wafers for the same device are produced, each with iteratively varying parameters (e.g. stack or component dimensions, layer materials, etc.) to be optimized. This usually results in dozens to hundreds of device measurements and corresponding data files, as statistics are collected. Scripting offers a way to automate repetitive but necessary tasks such as tidying, consolidation, pruning (i.e. outlier or failed device removal), numerical operations, plotting, and producing summary tables.

Engineers who are assigned data analysis duties typically already possess some programming experience. However, they may be inexperienced in coding for the aforementioned tasks. A useful strategy for learners is to acquire existing scripts from experienced colleagues or other projects, which may or may not be related to the project(s) in question, but nevertheless contain code to execute the relevant data processing. In the course of adapting these scripts to suit required purposes, learners develop the important scripting skills. 


\subsection{Parameter Extraction}

From raw data, important parameters need to be extracted. In the case of our DUT, $f_{3 \mathrm{~dB}}$ can be obtained from $S_{21}$ data. Extraction involves mathematical and numerical processing steps including smoothing, converting complex quantities to magnitude and phase, converting linear quantities to $\mathrm{dB}$, applying polynomial fits, normalization, and finding the $f_{3 \mathrm{~dB}}$ point. Besides $f_{3 \mathrm{~dB}}$, other parameters may also be of interest, such as device capacitance and resistance which can be calculated from $S_{22}$ data.

For the uninitiated, when and how to apply these operations, especially optional ones like polynomial fitting, might seem foreign. Coaching from experienced colleagues is therefore imperative.

\subsection{Discovering Trends}

Once relevant parameters have been extracted, the next step is to compare results from wafers possessing design and/or process differences. For example, the bandwidth can be compared across photodetector sizes. Data visualization is a powerful tool in this regard, and engineers need to be aware of what options exist and when to use them. In the example, comparative box plots are useful to determine the optimum detector dimensions for a given $f_{3 \mathrm{~dB}}$ requirement. On the other hand, when comparing device capacitances across different wafer process changes, a scatter plot may be more appriopriate.

Engineers normally learn these skills by self-reading and also observing their experienced colleagues and applying what they observed to their own analyses.

\section{CONCLUSIONS}

We have described industry-oriented training on on-wafer vector network analysis, using the example of an optoelectronic detector. We identified a general training methodology, then outlined various laboratory tasks and data analysis skills that operators and engineers should learn and master. The procedures and ideas pertaining to many tasks can be generalized. For example, Sec. 4.2.2 can be adapted to any VNA configuration (not just O/E). Moreover, the analysis skills in Sec. 5 can apply to contexts beyond integrated photonics.

We hope that this paper will be a useful reference to other organizations facing similar needs.

\section{REFERENCES}

[1] Davis, W. A. and Overstreet, W. P., "S parameters: A practical education," IEEE Trans. Educ. 32, 18-24 (Feb. 1989).

[2] Mulholland, J. E. and Noble, S. P., "An automated microwave measurements experience for undergraduates," IEEE Trans. Educ. 37, 147-150 (May 1994).

[3] Sadiku, M. N. O., "Deficiencies in the way scattering parameters are taught," IEEE Trans. Educ. 46, 399-404 (Aug. 2003).

[4] Karakekes, M. and Currier, D., "Optics education for machine operators in the semiconductor industry: moving beyond button pushing," in [Int. Conf. Educ. Opt.], Proc. SPIE 2525, 545-552 (1995).

[5] Moreno, R. and Mayer, R. E., "Cognitive principles of multimedia learning: The role of modality and contiguity," $J$. Educ. Psychol. 91 (2), 358-368 (1999).

[6] Paivio, A., [Mental Representations: A dual coding approach], Oxford University Press, New York, NY (1990).

[7] Fleming, N. D. and Mills, C., "Not another inventory, rather a catalyst for reflection," To Improve the Academy 11 (1), 137-155 (1992).

[8] Ludwig, R. and Bretchko, P., [RF Circuit Design: Theory and Applications], Prentice Hall, Upper Saddle River, NJ (2000).

[9] Lee, J., "Modeling of SOL calibration standards for PCB channel probing," IEEE Electrmagn. Compat. Mag. 5(4), 123-127 (2016).

[10] Bauer, R. F. and Penfield, P., “De-embedding and unterminating," IEEE Trans. Microw. Theory Techn. 22, 282-288 (Mar. 1974).

[11] Kruppa, W. and Sodomsky, K. F., "An explicit solution for the scattering parameters of a linear two-port measured with an imperfect test set (Correspondence)," IEEE Trans. Microw. Theory Techn. 19, 122-123 (Jan. 1971). 